$11-1-2013$

\title{
Parameter Estimations Based On Kumaraswamy Progressive Type II Censored Data with Random Removals
}

\author{
Navid Feroze \\ Government Post Graduate College Muzaffarabad, Azad Kashmir, Pakistan, navidferoz@hotmail.com \\ Ibrahim El-Batal \\ Cairo University, Cairo, Egypt, i_elbatal@yahoo.com
}

Follow this and additional works at: http://digitalcommons.wayne.edu/jmasm

Part of the Applied Statistics Commons, Social and Behavioral Sciences Commons, and the Statistical Theory Commons

\section{Recommended Citation}

Feroze, Navid and El-Batal, Ibrahim (2013) "Parameter Estimations Based On Kumaraswamy Progressive Type II Censored Data with Random Removals," Journal of Modern Applied Statistical Methods: Vol. 12 : Iss. 2 , Article 19.

DOI: $10.22237 /$ jmasm/1383279480

Available at: http://digitalcommons.wayne.edu/jmasm/vol12/iss2/19

This Regular Article is brought to you for free and open access by the Open Access Journals at DigitalCommons@WayneState. It has been accepted for inclusion in Journal of Modern Applied Statistical Methods by an authorized editor of DigitalCommons@WayneState. 


\section{Parameter Estimations Based On Kumaraswamy Progressive Type II Censored Data with Random Removals}

\author{
Navid Feroze \\ Government Post Graduate College \\ Muzaffarabad \\ Azad Kashmir, Pakistan
}

\author{
Ibrahim El-Batal \\ Cairo University \\ Cairo, Egypt
}

The estimation of two parameters of the Kumaraswamy distribution is considered under Type II progressive censoring with random removals, where the number of units removed at each failure time has a binomial distribution. The $M L E$ was used to obtain the estimators of the unknown parameters, and the asymptotic variance - covariance matrix was also obtained. The formula to compute the expected test time was derived. A numerical study was carried out for different combinations of model parameters. Different censoring schemes were used for the estimation, and performance of these schemes was compared.

Keywords: $\quad$ Expected test time, maximum likelihood estimation, progressive censoring, random removals

\section{Introduction}

Life tests are often one of the main research topics in many experimental designs. There are several situations in life testing, in reliability experiments and survival analysis in which units are lost or removed from the experiments while they are still alive. The loss may occur out of control or be reassigned. The out of control case can happen when an individual under study (testing) drops out. The other case may occur because of limitation of funds or to save time. For more details Balakrishnan and Aggarwala (2000) provide a comprehensive reference on the subject of progressive censoring and its applications. There are several types of censoring schemes; the Type II censoring scheme is one of the most common for consideration. In a Type II censoring, a total of $n$ units are placed on test, but

Navid Feroze is a lecturer at the Government Post Graduate College Muzaffarabad, Azad Kashmir, Pakistan.Email him at: navidferoz@hotmail.com.Dr.El-Batal is a professor in the Institute of Statistical Studies and Research, Department of Mathematical Statistics.Email him at i_elbatal@yahoo.com. 
instead of containing until all $n$ units have failed, the test is terminated at time of the $m_{\text {th }}(1 \leq m \leq n)$ unit failure. Type II censoring with different failure time distributions has been studied by many authors including Mann (1971), Meeker and Escobar (1991), and Lawless (2003).

If an experimenter desires to remove live units at points other than the $n^{\text {th }}$ termination point of the life test, the above described scheme will not be of use to the experimenter. Type II censoring does not allow for units to be lost or removed from the test at other than the $n_{\text {th }}$ termination point. This allowance may be desirable, as in the case of accidental breakage of experimental units, in which the loss of test units at points other than the termination point may be unavoidable. Intermediate removal may also be desirable when a compromise between reduced time of experimentation and the observation of at least some extreme lifetimes is sought. These reasons lead directly into the area of progressive censoring; see Balakrishnan and Agarwala (2000).

A generalization of Type II censoring is progressive Type II censoring. The progressive Type II censored life test is described as follows. Firstly, the experimenter places $n$ units on a test at time zero, with $m$ failures to be observed. When the first failure is observed, $r_{1}$ of the surviving units are randomly selected and removed. At the second observed failure, $r_{\text {th }}$ of the surviving units are randomly selected and removed. This experiment terminates at the time when the $m_{\text {th }}$ failure is observed and the remaining $r_{m}=n-r_{1}-\ldots-r_{m-1}-m$ surviving units are all removed. The statistical inference on the parameters of failure time distributions under progressive Type II censoring has been studied by several authors, such as Cohen (1963), Mann (1971), Viveros and Balakrishnan (1974), Balakrishnan and Aggarwala (2000), Ng et al. (2002), Chan and Balakrishnan (2004), Soliman (2008) and Raqab et al. (2010) (and the references therein). Note that, in this scheme, $r_{1}, r_{2}, \ldots, r_{m}$ are all pre-fixed. However, in some practical situations, these numbers may occur at random. Yuen and Tse (1996) indicated that, for example, the number of patients who drop out from a clinical test at each stage is random and cannot be pre-determined. In some reliability experiments, an experimenter may decide that it is inappropriate or too dangerous to carry on the testing on some of the tested units even though these units have not failed. In these cases, the pattern of removal at each failure is random. Suppose that any test unit being dropped out from the life test is independent of the others but with the same removal probability $p$. Then, Tse et al. (2004) indicated that the number of test units removed at each failure time has a binomial distribution. The main purpose of this article is to assess the required time to complete a life test under progressive Type II censored data with random removal (PCR). Assume that the 


\section{PARAMETER ESTIMATIONS BASED ON KUMARASWAMY DATA}

lifetime follows the Kumaraswamy distribution. The number of units removed at each failure time follows a binomial distribution with parameters $n$ and $p$.

\section{The Model}

The maximum likelihood estimators for the parameters of the Kumaraswamy distribution are derived based on progressive Type II censoring. Let random variable $X$ have a Kumaraswamy distribution with two positive shape parameters $\alpha$ and $\theta$. The probability density function of $X$ is given by

$$
f(x, \alpha, \theta)=\alpha \theta x^{\alpha-1}\left(1-x^{\alpha}\right)^{\theta-1}, 0<x<1, \alpha, \theta>0
$$

while the cumulative distribution function is given by

$$
F(x, \alpha, \theta)=1-\left(1-x^{\alpha}\right)^{\theta}, 0<x<1, \alpha, \theta>0
$$

Kumaraswamy (1980) was interested in distributions for hydrological random variables and actually proposed a mixture of a probability mass, at zero and density (1) over $(0,1)$.

The corresponding survival function of random variable $X$ is

$$
\bar{F}(x, \alpha, \theta)=\left(1-x^{\alpha}\right)^{\theta}, 0<x<1, \alpha, \theta>0
$$

and the failure (hazard) rate function takes the following form

$$
h(x)=\frac{\alpha \theta x^{\alpha-1}\left(1-x^{\alpha}\right)^{\theta-1}}{\left(1-x^{\alpha}\right)^{\theta}}=\frac{\alpha \theta x^{\alpha-1}}{\left(1-x^{\alpha}\right)}
$$

For $X \geq 0$, let $X_{1}<X_{2}<\ldots<X_{m}$ be the $m$ ordered failure times out of $n$ randomly selected items, where $m$ is predetermined before testing. At the $i_{\text {th }}$ failure, $r_{i}$ items are removed from the test. For progressive Type II censored sample with predetermined number of removals, say $R_{1}=r_{1}, R_{2}=r_{2}, \ldots . ., R_{m-1}=r_{m-1}$, where $R=R_{1}=r_{1}, R_{2}=r_{2}, \ldots . ., R_{m-1}=r_{m-1}$. 
Let $\left(X_{1}, X_{2}, \ldots, X_{m}\right)$ denote a progressive Type II censored sample. Then the joint probability density function of all $m$ progressive Type II censored order statistic is given by

$$
\begin{aligned}
f_{\mathrm{x}_{1}, \mathrm{x}_{2}, \ldots, \mathrm{x}_{m}} & \left(x_{1}, x_{2}, \ldots, x_{m}\right)=C \prod_{i=1}^{m} f\left(x_{i}\right)\left[1-F\left(x_{i}\right)\right]^{r_{i}} \\
& , x_{1}<x_{2}<\ldots,<x_{m} \\
\text { where } \quad & C=n\left(n-r_{1}-1\right)\left(n-r_{1}-r_{2}-1\right) \\
& \ldots\left(n-r_{1}-r_{2}-r_{3}-\ldots r_{m-1}-m+1\right)
\end{aligned}
$$

Thus for progressive Type II censoring with pre-determined number of removals $R=r$, the conditional likelihood function can be written as (Cohen, 1963)

$$
\begin{gathered}
L_{1}(x, \alpha, \theta \mid \mathrm{R}=\mathrm{r})=C \prod_{i=1}^{m} f\left(x_{i}\right)\left[1-F\left(x_{i}\right)\right]^{r_{i}}=C \prod_{i=1}^{m}\left[\alpha \theta x_{(i)}^{\alpha-1}\left(1-x_{(i)}^{\alpha}\right)^{\theta-1}\right] \\
{\left[\left(1-x_{(i)}^{\alpha}\right)\right]^{\gamma_{i}}}
\end{gathered}
$$

Equation (6) is derived conditional on $r_{i}$, where $r_{i}$ can be of any integer value between 0 and $n-m-\left(r_{1}+r_{2}+\ldots+r_{m-1}\right)$. The main difference between Type II progressive censoring and $P C R$ is that the $R$ are pre-determined in the former case while they are random in the latter case. Note that $m$ is predetermined in both cases. Under PCR, the $r_{i}$ terms are random. In particular, assume that each $r_{i}$ follows a binomial distribution, such that

$$
P\left(R_{1}=r_{1}\right)=\left(\begin{array}{c}
n-m \\
r_{1}
\end{array}\right) p^{r_{1}}(1-p)^{n-m-r_{1}}
$$

and

$$
\begin{aligned}
P\left(R_{i}=r_{i}, R_{i-1}=r_{i-1}, \ldots, R_{1}=r_{1}\right) & =\left(n-m-\sum_{j=1}^{i-1} r_{j}\right) \\
& \times p^{r_{i}}(1-p)^{n-m-\sum_{j=1}^{i-1}} r_{j}
\end{aligned}
$$




\section{PARAMETER ESTIMATIONS BASED ON KUMARASWAMY DATA}

where $0 \leq r_{i} \leq n-m-\left(r_{1}+r_{2}+\ldots+r_{m-1}\right)$.

Furthermore, assume that $R_{i}$ independent of $X_{i}$ for all $i$. Then the likelihood function can be found as

$$
L(x, \alpha, \theta, p \mid R=r)=L_{1}(x, \alpha, \theta \mid R=r) P(R, p)
$$

where $P(R, p)$ is the is the probability distribution of the $R$ terms $\left(R=r_{1}, r_{2}, \ldots\right.$, $\left.r_{m}\right)$ and, in particular, results in

$$
\begin{aligned}
P(R, p)= & P\left(R_{m-1}=r_{m-1} \mid R_{m-2}=r_{m-2}, \ldots, R_{1}=r_{1}\right) \\
& \times P\left(R_{m-2}=r_{m-2} \mid R_{m-3}=r_{m-3}, \ldots, R_{1}=r_{1}\right) \\
& \times P\left(R_{2}=r_{2} \mid R_{1}=r_{1}\right) P\left(R_{1}=r_{1}\right)
\end{aligned}
$$

Substituting (4) and (5) into (7) results in

$$
\begin{aligned}
P(R, p) & =\frac{(n-m) !}{\Pi_{i=1}^{m-1} r_{i} !\left(n-m-\sum_{j=1}^{i-1} r_{j}\right) !} p^{\sum_{i=1}^{m-1} r_{i}} \\
& \times(1-p)^{(m-1)(n-m)-\sum_{i=1}^{m-1}(m-i) r_{i}}
\end{aligned}
$$

and

$$
\begin{aligned}
\log P(R, p)= & C+\sum_{i=1}^{m-1} r_{i} \log p \\
& +\left[(m-1)(n-m)-\sum_{i=1}^{m-1}(m-i) r_{i}\right] \log (1-p)
\end{aligned}
$$

\section{Maximum Likelihood Estimation}

The maximum likelihood estimators of the parameters $\alpha, \theta$, and $p$ are derived based on progressive Type II censored data with binomial removals. Both point and interval estimations of the parameters are derived. 


\section{Point Estimations}

Because $P(R, p)$ does not depend on the parameters $\alpha$ and $\theta$, the maximum likelihood estimators (MLEs) of $\alpha$ and $\theta$ can be derived by maximizing (6) directly. Similarly, because $L_{1}(x, \alpha, \theta \mid R=r)$ does not involve the binomial parameter $p$, then the $M L E$ of $p$ can be found by maximizing $P(R, p)$ directly. The log likelihood function of (9) is given by

$$
\begin{aligned}
\log L(x, \alpha, \theta, p \mid R=r)= & \log L_{1}(x, \alpha, \theta \mid R=r)+\log P(R, p) \\
\text { where } & \\
\log L_{1}(x, \alpha, \theta \mid R=r)= & \log C+m \log \alpha+m \log \theta \\
& +(\alpha-1) \sum_{i=1}^{m} \log x_{(i)} \\
& +(\theta-1) \sum_{i=1}^{m} \log \left(1-x_{(i)}^{\alpha}\right)+\theta \sum_{i=1}^{m} r_{i} \log \left(1-x_{(i)}^{\alpha}\right)
\end{aligned}
$$

Take the partial derivative of $\log L_{1}(x, \alpha, \theta \mid R=r)$ with respect to $\alpha$ and $\theta$ and let them be zero

$$
\begin{aligned}
& \frac{\partial \log \left(L_{1}\right)}{\partial \alpha}=\frac{m}{\alpha}+m \log \theta+\sum_{i=1}^{m} \log x_{(i)}-(\theta-1) \sum_{i=1}^{m} \frac{x_{(i)}^{\alpha} \log x_{(i)}}{\left(1-x_{(i)}^{\alpha}\right)} \\
& -\theta \sum_{i=1}^{m} \frac{r_{i} x_{(i)}^{\alpha} \log x_{(i)}}{\left(1-x_{(i)}^{\alpha}\right)}=0 \\
& \text { and } \frac{\partial \log \left(L_{1}\right)}{\partial \theta}=\frac{m}{\theta}+\sum_{i=1}^{m} \log \left(1-x_{(i)}^{\alpha}\right)+\sum_{i=1}^{m} r_{i} \log \left(1-x_{(i)}^{\alpha}\right)=0
\end{aligned}
$$

$\widehat{\theta}=\frac{-m}{\sum_{i=1}^{m}\left(r_{i}+1\right) \log \left(1-x_{(i)}^{\alpha}\right)}$ is given by $\hat{\theta}$ of $\theta$. Thus the MLE $\hat{\alpha}$ of $\alpha$ and the $M L E$ is the numerical solution of equation (14).

It is observed from (14) that the MLE of the parameter $\alpha$ cannot be obtained in closed form. It can be obtained by solving a one dimensional optimization problem. A simple fixed point iteration algorithm can be used to solve this 


\section{PARAMETER ESTIMATIONS BASED ON KUMARASWAMY DATA}

optimization problem. Firstly, the parameter $\theta$ in log-likelihood (13) has been replaced by its $M L E$ the $\hat{\boldsymbol{\theta}}$ resultant log-likelihood becomes

$$
\begin{aligned}
& \log L_{1}(x, \alpha, \theta \mid \mathrm{R}=\mathrm{r})=\log C+m \log \alpha+m \log \left\{\frac{-m}{\sum_{i=1}^{m}\left(r_{i}+1\right) \log \left(1-x_{(i)}^{\alpha}\right)}\right\} \\
& +(\alpha-1) \sum_{i=1}^{m} \log x_{(i)}+\left\{\frac{-m}{\sum_{i=1}^{m}\left(r_{i}+1\right) \log \left(1-x_{(i)}^{\alpha}\right)}-1\right\} \sum_{i=1}^{m} \log \left(1-x_{(i)}^{\alpha}\right) \\
& +\left\{\frac{-m}{\sum_{i=1}^{m}\left(r_{i}+1\right) \log \left(1-x_{(i)}^{\alpha}\right)}\right\} \sum_{i=1}^{m} r_{i} \log \left(1-x_{(i)}^{\alpha}\right)
\end{aligned}
$$

After some simplification it can be presented as

$$
\begin{gathered}
\log L_{1}(x, \alpha \mid R=r) \propto m \log \alpha+m \log \left\{\sum_{i=1}^{m}\left(r_{i}+1\right) \log \left(1-x_{(i)}^{\alpha}\right)\right\} \\
+(\alpha-1) \sum_{i=1}^{m} \log x_{(i)}-\sum_{i=1}^{m}\left(r_{i}+1\right) \log \left(1-x_{(i)}^{\alpha}\right)
\end{gathered}
$$

$M L E$ of $\alpha$ can be obtained by maximizing (15) with respect to $\alpha$ and it is unique. Most of the standard iterative process can be used for finding the MLE. The following simple algorithm is proposed: If $\hat{\alpha}$ is the MLE of $\alpha$, then it is obvious from $l(\alpha)=\frac{\partial \log L_{1}(x, \alpha \mid R=r)}{\partial \alpha}=0$ that $\hat{\alpha}$ satisfies the following fixed point type equation; $g(\alpha)=\alpha$ 


$$
\begin{aligned}
\frac{\partial \log L_{1}(x, \alpha \mid R=r)}{\partial \alpha}= & \frac{m}{\alpha}-\frac{m \sum_{i=1}^{m} \frac{\left(r_{i}+1\right) x_{(i)}^{\alpha} \log x_{(i)}}{\left(1-x_{(i)}^{\alpha}\right)}}{\sum_{i=1}^{m}\left(r_{i}+1\right) \log \left(1-x_{(i)}^{\alpha}\right)} \\
& +\sum_{i=1}^{m} \log x_{(i)}-\sum_{i=1}^{m} \frac{\left(r_{i}+1\right) x_{(i)}^{\alpha} \log x_{(i)}}{\left(1-x_{(i)}^{\alpha}\right)}=0
\end{aligned}
$$

where

$$
\alpha=g(\alpha)=\left[\frac{1}{m}\left\{\sum_{i=1}^{m} \frac{\left(r_{i}+1\right) x_{(i)}^{\alpha} \log x_{(i)}}{\left(1-x_{(i)}^{\alpha}\right)}-\sum_{i=1}^{m} \log x_{(i)}\right\}+\frac{\sum_{i=1}^{m} \frac{\left(r_{i}+1\right) x_{(i)}^{\alpha} \log x_{(i)}}{\left(1-x_{(i)}^{\alpha}\right)}}{\sum_{i=1}^{m}\left(r_{i}+1\right) \log \left(1-x_{(i)}^{\alpha}\right)}\right]^{-1}
$$

The iterated result of the above function has been considered as an $M L E$ of $\alpha$ and denoted by $\hat{\alpha}$. Now the approximate MLE of $\alpha$ has been incorporated in (14) to obtain the $M L E$ of $\beta$.

Similarly, from (12) the partial derivative of $\log P(R, p)$ with respect to binomial parameter $p$ can be obtained by solving the following equation

$$
\frac{\partial \log P(p, R)}{\partial p}=\frac{\sum_{i=1}^{m-1} r_{i}}{p}-\frac{(m-1)(n-m)-\sum_{i=1}^{m-1}(m-i) r_{i}}{1-p}=0
$$

thus the MLE of $\hat{p}$ of $p$ is given by

$$
\widehat{p}=\frac{\sum_{i=1}^{m-1} r_{i}}{(m-1)(n-m)-\sum_{i=1}^{m-1}(m-i-1) r_{i}}
$$

\section{Interval Estimations}

The approximate confidence intervals of the parameters based on the asymptotic distributions of the MLE of the parameters $\alpha, \theta$ and $p$ are derived in this subsection. The elements of the Fisher information matrix for the parameters of 
the Kumaraswamy distribution based on progressive censored samples have been derived explicitly. The Fisher information matrix can be defined as

$$
\begin{aligned}
I(\alpha, \theta, p) & =-\mathrm{E}\left[\begin{array}{ccc}
\frac{\partial^{2} \log (L)}{\partial \alpha^{2}} & \frac{\partial^{2} \log (L)}{\partial \alpha \partial \theta} & 0 \\
\frac{\partial^{2} \log (L)}{\partial \theta \partial \alpha} & \frac{\partial^{2} \log (L)}{\partial \theta^{2}} & 0 \\
0 & 0 & \frac{\partial^{2} \log (L)}{\partial p^{2}}
\end{array}\right] \\
& =\left[\begin{array}{ccc}
I_{\alpha \alpha} & I_{\alpha \theta} & 0 \\
I_{\theta \alpha} & I_{\theta \theta} & 0 \\
0 & 0 & I_{p p}
\end{array}\right]
\end{aligned}
$$

For the information matrix for $\alpha, \theta$ and $p$, find

$$
\begin{aligned}
& \frac{-\partial^{2} \log (L)}{\partial \alpha^{2}}= \frac{m}{\alpha^{2}}+\sum_{i=1}^{m} \frac{x_{(i)}^{\alpha}\left(\log x_{(i)}\right)^{2}}{\left(1-x_{(i)}^{\alpha}\right)^{2}}\left(\theta+\theta r_{i}-1\right), \\
& \frac{-\partial^{2} \log (L)}{\partial \theta^{2}}=\frac{m}{\theta^{2}} \\
& \frac{-\partial^{2} \log (L)}{\partial \alpha \partial \theta}=\sum_{i=1}^{m} \frac{x_{(i)}^{\alpha} \log x_{(i)}}{\left(1-x_{(i)}^{\alpha}\right)}+\sum_{i=1}^{m} \frac{r_{i} x_{(i)}^{\alpha} \log x_{(i)}}{\left(1-x_{(i)}^{\alpha}\right)} \\
& \frac{-\partial^{2} \log (L)}{\partial P^{2}}=\frac{\sum_{I=}^{m-1} r_{i}}{p^{2}}+\frac{(m-1)(n-m)-\sum_{i=1}^{m-1}(m-i) r_{i}}{(1-p)^{2}},
\end{aligned}
$$

and

$$
\frac{-\partial^{2} \log (L)}{\partial \alpha \partial p}=\frac{-\partial^{2} \log (L)}{\partial \theta \partial p}=0
$$


In order to derive the expressions for $I_{\alpha \alpha}, I_{\alpha \theta}, I_{\theta \theta}$ and $I_{p p}$ the distribution of the $i^{\text {th }}$ order statistics from the Kumaraswamy distribution is required, which can be written as

$$
\begin{aligned}
g\left(x_{(i)}\right)= & C_{n \bar{f}} \alpha \theta x_{(i)}^{\alpha-1}\left(1-x_{(i)}^{\alpha}\right)^{\theta-1} \\
& \times\left\{1-\left(1-x_{(i)}^{\alpha}\right)^{\theta}\right\}^{i-1}\left(1-x_{(i)}^{\alpha}\right)^{-\theta(n-i)}, 0<x_{(i)}<1
\end{aligned}
$$

where $C_{n, i}=\frac{n !}{(i-1) !(n-i) !}$

Here, the expectations necessary to derive the elements of the Fisher information matrix are

$$
\begin{aligned}
& \mathrm{E}\left[\frac{x_{(i)}^{\alpha} \log x_{(i)}}{\left(1-x_{(i)}^{\alpha}\right)}\right]=C_{n, i} \alpha \theta \int_{0}^{1} \log x_{(i)} x_{(i)}^{2 \alpha-1}\left(1-x_{(i)}^{\alpha}\right)^{\theta(n+1-\bar{i})-2}\left\{1-\left(1-x_{(i)}^{\alpha}\right)^{\theta}\right\}^{i-1} d x_{(i)} \\
& =C_{n, i} \alpha \theta \sum_{j=0}^{i-1}(-1)^{j}\left(\begin{array}{c}
i-1 \\
j
\end{array}\right) \int_{0}^{1} \log x_{(i)} x_{(i)}^{2 \alpha-1}\left(1-x_{(i)}^{\alpha}\right)^{\theta(n+1-i+j)-2} d x_{(i)} \\
& =C_{n, i} \alpha \theta \sum_{j=0}^{i-1}(-1)^{j}\left(\begin{array}{c}
i-1 \\
j
\end{array}\right) \frac{1-\text { HarmanicNumber }[\theta(n+1-i+j)]}{4 \alpha^{2} \theta(n+1-i+j)\{\theta(n+1-i+j)-1\}} \\
& \mathrm{E}\left[\frac{x_{(i)}^{\alpha}\left(\log x_{(i)}\right)^{2}}{\left(1-x_{(i)}^{\alpha}\right)^{2}}\right]=C_{n, i} \alpha \theta \sum_{j=0}^{i-1}(-1)^{j}\left(\begin{array}{c}
i-1 \\
j
\end{array}\right)_{0}^{1}\left(\log x_{(i)}\right)^{2} x_{(i)}^{2 \alpha-1}\left(1-x_{(i)}^{\alpha}\right)^{\theta(n+1-i+j)-3} d x_{(i)} \\
& 6(\gamma-2) \gamma \\
& +\pi^{2} \\
& +6 \psi(0, \theta(n+1-i+j))\{2 \gamma+\psi(0, \theta(n+1-i+j))-2\} \\
& =C_{n, i} \alpha \theta \sum_{j=0}^{i-1}(-1)^{j}\left(\begin{array}{c}
i-1 \\
j
\end{array}\right) \frac{-6 \psi(1, \theta(n+1-i+j))}{48 \alpha^{3}\{\theta(n+1-i+j)-1\}\{\theta(n+1-i+j)-2\}}
\end{aligned}
$$

where $\gamma$ and $\psi(a, b)$ are Euler gamma and Poly gamma functions respectively. Using these results, the Fisher information matrix can be obtained, which can 


\section{PARAMETER ESTIMATIONS BASED ON KUMARASWAMY DATA}

further be used to derive the elements of the approximate variance-covariance matrix as

$$
V=\left(\begin{array}{lll}
V_{\alpha \alpha} & V_{\alpha \theta} & 0 \\
V_{\theta \alpha} & V_{\theta \theta} & 0 \\
0 & 0 & V_{p p}
\end{array}\right)=\left(\begin{array}{lll}
I_{\alpha \alpha} & I_{\alpha \theta} & 0 \\
I_{\theta \alpha} & I_{\theta \theta} & 0 \\
0 & 0 & I_{p p}
\end{array}\right)^{-1}
$$

$\widehat{\alpha}, \hat{\theta}, \hat{p}$. It is known that the asymptotic distribution of the $M L E$ is given by

$$
\left(\begin{array}{l}
\hat{\alpha} \\
\hat{\theta} \\
\hat{p}
\end{array}\right) \approx N\left[\left(\begin{array}{l}
\alpha \\
\theta \\
p
\end{array}\right),\left(\begin{array}{lll}
V_{\alpha \alpha} & V_{\alpha \theta} & 0 \\
V_{\theta \alpha} & V_{\theta \theta} & 0 \\
0 & 0 & V_{p p}
\end{array}\right)\right]
$$

Because $V$ involves the parameters $\alpha, \theta$ and $p$, replace the parameters by the corresponding maximum likelihood estimators in order to obtain an estimate of $V$, which is denoted by $\hat{V}$. Using (18), approximate $100(\gamma) \%$ confidence intervals for

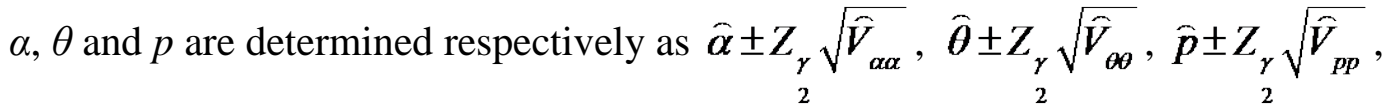
where $z_{\gamma}$ is the upper $100(\gamma) \%$ percentile of the standard normal distribution.

\section{The Expected Time Test}

In practical applications, it is often useful to have an idea of the test time of the whole test. For progressive Type II censoring sampling plan with random or binomial removals, the expected test time for the experiment is given by the expectation of the $m^{\text {th }}$ order statistic $X_{(m)}$. From Balakrishnan and Aggarwala (2000), the conditional expectation of $X_{(m)}$ for a fixed set of $R=R_{1}=r_{1}, R_{2}=r_{2}$, $R_{m-1}=r_{m-1}$ is given by

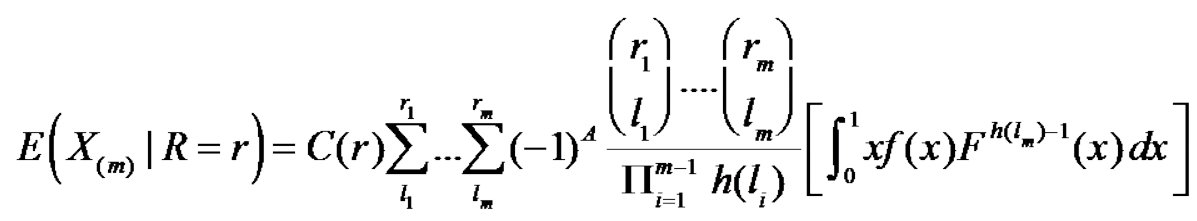


where

$$
A=\sum_{i=1}^{m} l_{i}, C(r)=n\left(n-r_{1}-1\right)\left(n-r_{1}-r_{2}-2\right) \ldots\left(n-\sum_{i=1}^{m-1}\left(r_{i}+1\right)\right), h(l)=l_{1}+l_{2}+l_{i}+i
$$

and ' $i$ ' is the number of live units removed from experimentation (or number of failure units). Substituting (1) and (2) into (19) results in the following

$$
\begin{aligned}
E\left(X_{(m)} \mid R=r\right)= & C(r) \sum_{l_{1}}^{r_{1}} \ldots \sum_{l_{m}}^{r_{m}}(-1)^{A} \frac{\left(\begin{array}{l}
r_{1} \\
l_{1}
\end{array}\right) \cdots\left(\begin{array}{l}
r_{m} \\
l_{m}
\end{array}\right)}{\prod_{i=1}^{m-1} h\left(l_{i}\right)}\left[\int_{0}^{1} x \alpha \theta x^{\alpha-1}\left(1-x^{\alpha}\right)^{\theta-1}\right] \\
& \times\left[1-\left(1-x^{\alpha}\right)^{\theta}\right]^{h\left(l_{m}\right)-1} d x
\end{aligned}
$$

Let

$$
\begin{aligned}
S & =\left[\int_{0}^{1} x \alpha \theta x^{\alpha-1}\left(1-x^{\alpha}\right)^{\theta-1}\right]\left[1-\left(1-x^{\alpha}\right)^{\theta}\right]^{h\left(l_{m}\right)-1} d x \\
& =\int_{0}^{1} x \alpha \theta x^{\alpha-1}\left(1-x^{\alpha}\right)^{\theta-1} \sum_{k=0}^{h\left(l_{m}\right)-1}(-1)^{k}\left(\begin{array}{c}
h\left(l_{m}\right)-1 \\
k
\end{array}\right)\left(1-x^{\alpha}\right)^{\theta k} d x \\
& =\theta \alpha \sum_{k=0}^{h\left(l_{m}\right)-1}(-1)^{k}\left(\begin{array}{c}
\left.h\left(l_{m}\right)-1\right) \\
k
\end{array}\right) \int_{0}^{1} x^{\alpha}\left(1-x^{\alpha}\right)^{\theta k+\theta-1} d x \\
& =\theta \sum_{k=0}^{h\left(l_{m}\right)-1}(-1)^{k}\left(\begin{array}{c}
h\left(l_{m}\right)-1 \\
k
\end{array}\right) \mathrm{B}\left(1+\frac{1}{\alpha}, \theta(k+1)\right)
\end{aligned}
$$

Plugging this quantity into the right hand side of equation (20), the expected test time of progressive Type II censoring with fixed number of removal will be

$$
\begin{aligned}
E\left(X_{(m)}\right. & \mid R=r)=\theta C(r) \sum_{l_{1}}^{r_{1}} \ldots \sum_{l_{m}}^{r_{m}}(-1)^{A} \frac{\left(\begin{array}{c}
r_{1} \\
l_{1}
\end{array}\right) \ldots\left(\begin{array}{l}
r_{m} \\
l_{m}
\end{array}\right)}{\Pi_{i=1}^{m-1} h\left(l_{i}\right)} \\
& \times \sum_{k=0}^{h\left(l_{m}\right)-1}(-1)^{k}\left(\begin{array}{c}
h\left(l_{m}\right)-1 \\
k
\end{array}\right) \mathrm{B}\left(1+\frac{1}{\alpha}, \theta(k+1)\right)
\end{aligned}
$$




\section{PARAMETER ESTIMATIONS BASED ON KUMARASWAMY DATA}

Also, the expected time under the Type II censoring scheme without removal is defined by the expected value of the $m_{\text {th }}$ failure time, denoted by $X^{*}(m)$ where

$$
E\left(X^{*}(m)\right)=m \theta\left(\begin{array}{l}
n \\
m
\end{array}\right) \sum_{k=0}^{m-1}(-1)^{k}\left(\begin{array}{c}
m-1 \\
k
\end{array}\right) \mathrm{B}\left(1+\frac{1}{\alpha}, \theta(k+1)\right)
$$

Because $r_{i}=0$ for all $i=1,2, \ldots, m-1$. Similarly, the expected value of $X_{(m)}$ for complete sample can be derived from (22) by setting $m=n$ and $r_{i}=0$ as

$$
E\left(X^{* *}(m)\right)=n \theta \sum_{k=0}^{m-1}(-1)^{k}\left(\begin{array}{c}
n-1 \\
k
\end{array}\right) \mathrm{B}\left(1+\frac{1}{\alpha}, \theta(k+1)\right)
$$

Under PCR, the $R$ terms are random. The expected time to complete an experiment under $P C R$ is given by taking the expectation of both sides equation (21) with respect to the $R$ terms. That is

$$
E\left[\left(X_{(m)}\right)\right]=E_{R} E\left[\left(X_{(m)}\right) \mid R=r\right]=\sum_{r_{1}=0}^{g\left(r_{1}\right)} \sum_{r_{2}=0}^{g\left(r_{2}\right)} \ldots \sum_{r_{m-1}=0}^{g\left(r_{m-1}\right)} P(R) E\left[\left(X_{(m)}\right) \mid R=r\right]
$$

where $g\left(r_{i}\right)=n-m-\left(r_{1}+r_{2}+\ldots+r_{i-1}\right)$ and $P(R)$ is given in (10). Thus equation (24) gives an expression to compute the expected time for given values of $m$ and $n$. To see how much time is saved under Type II progressive censoring, compare equations (23) and (24) where the ratio of the expected test time for Type I progressive censoring sample with binomial removals $(P C R)$ with respect to the expected time for complete sample , that is

$$
R E E T_{1}=\frac{E\left[\left(X_{(m)}\right)\right] \text { under PCR for a sample size } n}{E\left(X_{(m)}^{* *}\right) \text { under complete sampling for a sample size } n}
$$

If replacing the numerator by the expected test time under Type II progressive censoring with random removals $(P C R)$, this ratio is defined by $R E E T_{2}$. Notice that the ratios $R E E T_{1}$ and $R E E T_{2}$ provide important information in determining the shortest experiment time significantly if the sample size $n$ is large. When $R E E T_{1}$ and $R E E T_{2}$ are closer to one, the test time under respective 
censoring scheme is closer to the complete sample. The influence of the binomial probability removals $p$ on the expected time can be studied by analyzing $R E E T_{1}$ for various values of $p$. The comparisons between the three expected times will be made in order to reward some information about $m$ and $n$ on the duration of the experiment. As it seems, analytical comparisons between these three expected times is difficult. Therefore, these comparisons can be made numerically for various values of $m, n, \alpha$, and $\theta$.

\section{Numerical Study}

The MLEs, their variances and 95\% confidence intervals for parameters of the Kumaraswamy distribution using progressively censored data with random removals are now computed. The computations were made for different censoring schemes including various choices of $m$ and $n$. the parametric space includes $(\alpha, \theta) \in\left\{\begin{array}{l}(0.50,0.75),(1.00,0.80),(2.00,3.00), \\ (2.50,1.50),(5.00,3.00),(4.00,2.50)\end{array}\right\}$.

The censoring schemes are framed as follows:

\section{Scheme 1:}

$n=20, m=15$,

$r_{1}=\ldots=r_{14}=0$,

$r_{15}=5$

Scheme 4:

$n=20, m=18$,

$r_{1}=\ldots=r_{17}=0$,

$r_{18}=2$

Scheme 7:

$n=30, m=20$,

$r_{1}=\ldots=r_{19}=0$,

$r_{20}=10$

Scheme 10:

$n=30, m=25$,

$r_{1}=\ldots=r_{19}=0$,

$r_{20}=5$
Scheme 2:

$n=20, m=15$,

$r_{1}=\ldots=r_{7}=r_{9}=\ldots=r_{15}=0, \quad r_{2}=\ldots=r_{15}=0$,

$r_{8}=5$

Scheme 5:

$n=20, m=18$,

$r_{1}=\ldots=r_{8}=r_{11}=\ldots=r_{18}=0, \quad r_{2}=\ldots=r_{18}=0$,

$r_{9}=r_{10}=1$

Scheme 8:

$n=30, m=20$,

$r_{1}=\ldots=r_{10}=r_{13}=\ldots=r_{20}=0$,

$r_{11}=r_{12}=5$

Scheme 11:

$n=30, m=25$,

$r_{1}=\ldots=r_{10}=r_{13}=\ldots=r_{20}=0$,

$r_{11}=2, r_{12}=3$

\section{Scheme 3:}

$n=20, m=15$,

$r_{1}=5$

\section{Scheme 6:}

$n=20, m=18$,

$r_{1}=2$

\section{Scheme 9:}

$n=30, m=20$,

$r_{2}=\ldots=r_{20}=0$,

$r_{1}=10$

Scheme 12:

$n=30, m=25$,

$r_{2}=\ldots=r_{20}=0$,

$r_{1}=5$ 


\section{PARAMETER ESTIMATIONS BASED ON KUMARASWAMY DATA}

Scheme 13:

$n=40, m=30$,

$r_{1}=\ldots=r_{29}=0$,

$r_{30}=10$

Scheme 16:

$n=40, m=36$,

$r_{1}=\ldots=r_{35}=0$,

$r_{36}=4$
Scheme 14:

$n=40, m=30$,

$r_{15}=r_{16}=5$

Scheme 15:

$n=40, m=30$,

$r_{1}=10$

Scheme 17:

$n=40, m=36$,

$r_{1}=\ldots=r_{17}=r_{20}=\ldots=r_{36}=0$,

$r_{18}=r_{19}=2$
Scheme 18:

$n=40, m=36$,

$r_{2}=\ldots=r_{36}=0$,

$r_{1}=4$

The notations used in the tables are

$V(\hat{\alpha})$ : Variance of the estimator

$L L(\hat{\alpha})$ : Lower limit of the confidence interval

$U L(\hat{\alpha}):$ Upper limit of the confidence interval

Table 1. $M L E \mathrm{~s}$, their variances and $95 \%$ confidence intervals for parameters using $\alpha=0.50, \theta=0.75$

\begin{tabular}{ccccccccc}
\hline Schemes & $\hat{\boldsymbol{\alpha}}$ & $\hat{\boldsymbol{\theta}}$ & $\boldsymbol{V}(\hat{\boldsymbol{\alpha}})$ & $\boldsymbol{V}(\hat{\boldsymbol{\theta}})$ & $L L(\hat{\alpha})$ & $U L(\hat{\alpha})$ & $L L(\hat{\theta})$ & $U L(\hat{\theta})$ \\
\hline 1 & 0.674492 & 0.941582 & 0.088685 & 0.059105 & 0.090802 & 1.258182 & 0.465076 & 1.418088 \\
2 & 0.675560 & 0.955438 & 0.090411 & 0.060857 & 0.086219 & 1.264902 & 0.471920 & 1.438956 \\
3 & 0.689900 & 0.988406 & 0.096476 & 0.065130 & 0.081111 & 1.298689 & 0.488204 & 1.488608 \\
4 & 0.640946 & 0.917092 & 0.087820 & 0.046725 & 0.060112 & 1.221780 & 0.493417 & 1.340766 \\
5 & 0.640955 & 0.926610 & 0.088027 & 0.047700 & 0.059434 & 1.222475 & 0.498538 & 1.354682 \\
6 & 0.649568 & 0.934912 & 0.088397 & 0.048559 & 0.066829 & 1.232306 & 0.503005 & 1.366819 \\
7 & 0.615257 & 0.909485 & 0.073949 & 0.041358 & 0.082264 & 1.148250 & 0.510886 & 1.308085 \\
8 & 0.620614 & 0.912566 & 0.080909 & 0.041639 & 0.063103 & 1.178126 & 0.512616 & 1.312516 \\
9 & 0.632944 & 0.915290 & 0.084524 & 0.041888 & 0.063112 & 1.202776 & 0.514146 & 1.316433 \\
10 & 0.581573 & 0.836671 & 0.071226 & 0.028001 & 0.058485 & 1.104661 & 0.508696 & 1.164646 \\
11 & 0.584405 & 0.854518 & 0.072029 & 0.029208 & 0.058375 & 1.110434 & 0.519547 & 1.189490 \\
12 & 0.602121 & 0.896171 & 0.073575 & 0.032125 & 0.070475 & 1.133767 & 0.544872 & 1.247470 \\
13 & 0.546138 & 0.809125 & 0.058654 & 0.021823 & 0.071455 & 1.020821 & 0.519583 & 1.098667 \\
14 & 0.546197 & 0.816111 & 0.059933 & 0.022201 & 0.066365 & 1.026028 & 0.524069 & 1.108152 \\
15 & 0.553892 & 0.823240 & 0.064746 & 0.022591 & 0.055164 & 1.052621 & 0.528647 & 1.117833 \\
16 & 0.510985 & 0.774778 & 0.038303 & 0.016674 & 0.127392 & 0.894579 & 0.521684 & 1.027872 \\
17 & 0.532987 & 0.781340 & 0.042096 & 0.016958 & 0.130846 & 0.935128 & 0.526102 & 1.036577 \\
18 & 0.536114 & 0.796164 & 0.048681 & 0.017608 & 0.103665 & 0.968562 & 0.536084 & 1.056245 \\
\hline & & & & & & & &
\end{tabular}


FEROZE \& EL-BATAL

Table 2. MLEs, their variances and $95 \%$ confidence intervals for parameters using $\alpha=1.00, \theta=0.80$

\begin{tabular}{ccccccccc}
\hline Schemes & $\hat{\alpha}$ & $\hat{\boldsymbol{\theta}}$ & $V(\hat{\alpha})$ & $V(\hat{\boldsymbol{\theta}})$ & $L L(\hat{\boldsymbol{\alpha}})$ & $U L(\hat{\alpha})$ & $L L(\hat{\boldsymbol{\theta}})$ & $U L(\hat{\boldsymbol{\theta}})$ \\
\hline 1 & 1.232107 & 1.026742 & 0.103913 & 0.070280 & 0.600290 & 1.863925 & 0.507139 & 1.546344 \\
2 & 1.238510 & 1.026802 & 0.105153 & 0.070288 & 0.602936 & 1.874084 & 0.507168 & 1.546435 \\
3 & 1.242170 & 1.027064 & 0.106155 & 0.070324 & 0.603573 & 1.880767 & 0.507298 & 1.546830 \\
4 & 1.218273 & 0.985327 & 0.099675 & 0.053937 & 0.599476 & 1.837070 & 0.530129 & 1.440525 \\
5 & 1.220254 & 0.987677 & 0.099778 & 0.054195 & 0.601136 & 1.839372 & 0.531393 & 1.443960 \\
6 & 1.220848 & 1.008086 & 0.103164 & 0.056458 & 0.591313 & 1.850382 & 0.542374 & 1.473797 \\
7 & 1.174262 & 0.958474 & 0.095742 & 0.045934 & 0.567794 & 1.780731 & 0.538404 & 1.378543 \\
8 & 1.190808 & 0.961994 & 0.097742 & 0.046272 & 0.578039 & 1.803578 & 0.540381 & 1.383606 \\
9 & 1.215313 & 0.969807 & 0.098676 & 0.047026 & 0.599623 & 1.831002 & 0.544770 & 1.394844 \\
10 & 1.132875 & 0.937474 & 0.083493 & 0.035154 & 0.566531 & 1.699219 & 0.569984 & 1.304964 \\
11 & 1.141405 & 0.941570 & 0.089372 & 0.035462 & 0.555460 & 1.727350 & 0.572475 & 1.310666 \\
12 & 1.163471 & 0.942070 & 0.092902 & 0.035500 & 0.566065 & 1.760876 & 0.572779 & 1.311362 \\
13 & 1.113024 & 0.878943 & 0.080758 & 0.025751 & 0.556034 & 1.670015 & 0.564417 & 1.193469 \\
14 & 1.128151 & 0.928314 & 0.081152 & 0.028726 & 0.569802 & 1.686501 & 0.596121 & 1.260507 \\
15 & 1.129390 & 0.936065 & 0.081241 & 0.029207 & 0.570737 & 1.688044 & 0.601098 & 1.271031 \\
16 & 1.110531 & 0.853722 & 0.076051 & 0.020246 & 0.570015 & 1.651048 & 0.574840 & 1.132605 \\
17 & 1.111389 & 0.856941 & 0.078882 & 0.020399 & 0.560905 & 1.661872 & 0.577007 & 1.136875 \\
18 & 1.111504 & 0.865018 & 0.080522 & 0.020785 & 0.555328 & 1.667680 & 0.582445 & 1.147590 \\
\hline
\end{tabular}




\section{PARAMETER ESTIMATIONS BASED ON KUMARASWAMY DATA}

Table 3. MLEs, their variances and $95 \%$ confidence intervals for parameters using $\alpha=2.00, \theta=3.00$

\begin{tabular}{ccccccccc}
\hline Schemes & $\hat{\alpha}$ & $\hat{\boldsymbol{\theta}}$ & $V(\hat{\alpha})$ & $V(\hat{\boldsymbol{\theta}})$ & $L L(\hat{\alpha})$ & $U L(\hat{\alpha})$ & $L L(\hat{\boldsymbol{\theta}})$ & $U L(\hat{\boldsymbol{\theta}})$ \\
\hline 1 & 2.405773 & 3.331680 & 0.173545 & 0.740006 & 1.589262 & 3.222285 & 1.645617 & 5.017742 \\
2 & 2.420848 & 3.341618 & 0.175239 & 0.744427 & 1.600362 & 3.241334 & 1.650526 & 5.032709 \\
3 & 2.424628 & 3.349518 & 0.180851 & 0.747951 & 1.591108 & 3.258149 & 1.654428 & 5.044608 \\
4 & 2.363294 & 3.323579 & 0.153386 & 0.613677 & 1.595669 & 3.130919 & 1.788164 & 4.858994 \\
5 & 2.390266 & 3.328627 & 0.161481 & 0.615542 & 1.602645 & 3.177887 & 1.790880 & 4.866374 \\
6 & 2.395203 & 3.331073 & 0.172947 & 0.616447 & 1.580101 & 3.210306 & 1.792196 & 4.869950 \\
7 & 2.309179 & 3.258572 & 0.131193 & 0.530914 & 1.599256 & 3.019103 & 1.830440 & 4.686704 \\
8 & 2.313648 & 3.285888 & 0.133527 & 0.539853 & 1.597437 & 3.029859 & 1.845784 & 4.725992 \\
9 & 2.325209 & 3.321773 & 0.135464 & 0.551709 & 1.603822 & 3.046596 & 1.865942 & 4.777604 \\
10 & 2.254230 & 3.191529 & 0.110980 & 0.407434 & 1.601281 & 2.907178 & 1.940450 & 4.442609 \\
11 & 2.255714 & 3.220759 & 0.121270 & 0.414931 & 1.573166 & 2.938263 & 1.958221 & 4.483296 \\
12 & 2.278655 & 3.255155 & 0.125297 & 0.423841 & 1.584868 & 2.972441 & 1.979134 & 4.531176 \\
13 & 2.180235 & 3.120957 & 0.102417 & 0.324679 & 1.552983 & 2.807487 & 2.004137 & 4.237778 \\
14 & 2.184516 & 3.135531 & 0.102438 & 0.327719 & 1.557201 & 2.811832 & 2.013496 & 4.257567 \\
15 & 2.253760 & 3.140167 & 0.103276 & 0.328688 & 1.623882 & 2.883637 & 2.016473 & 4.263862 \\
16 & 2.104538 & 3.112679 & 0.082326 & 0.269133 & 1.542166 & 2.666911 & 2.095871 & 4.129487 \\
17 & 2.133270 & 3.117755 & 0.099195 & 0.270011 & 1.515963 & 2.750577 & 2.099288 & 4.136221 \\
18 & 2.177042 & 3.117986 & 0.100672 & 0.270051 & 1.555156 & 2.798928 & 2.099444 & 4.136528 \\
\hline
\end{tabular}




\section{FEROZE \& EL-BATAL}

Table 4. MLEs, their variances and $95 \%$ confidence intervals for parameters using $\alpha=2.50, \theta=1.50$

\begin{tabular}{|c|c|c|c|c|c|c|c|c|}
\hline Schemes & $\hat{\alpha}$ & $\hat{\boldsymbol{\theta}}$ & $V(\hat{\alpha})$ & $V(\hat{\theta})$ & $L L(\hat{\alpha})$ & $U L(\hat{\alpha})$ & $L L(\hat{\theta})$ & $U L(\hat{\theta})$ \\
\hline 1 & 2.747904 & 1.730365 & 0.239122 & 0.199611 & 1.789462 & 3.706346 & 0.854679 & 2.606050 \\
\hline 2 & 2.757010 & 1.732250 & 0.240154 & 0.200046 & 1.796503 & 3.717517 & 0.855610 & 2.608889 \\
\hline 3 & 2.792415 & 1.733128 & 0.241268 & 0.200249 & 1.829681 & 3.755148 & 0.856044 & 2.610212 \\
\hline 4 & 2.708039 & 1.681290 & 0.227382 & 0.157041 & 1.773420 & 3.642658 & 0.904574 & 2.458007 \\
\hline 5 & 2.735921 & 1.713229 & 0.228650 & 0.163064 & 1.798701 & 3.673141 & 0.921757 & 2.504700 \\
\hline 6 & 2.739513 & 1.728698 & 0.237295 & 0.166022 & 1.784740 & 3.694287 & 0.930080 & 2.527315 \\
\hline 7 & 2.704854 & 1.647548 & 0.191089 & 0.135721 & 1.848065 & 3.561643 & 0.925478 & 2.369617 \\
\hline 8 & 2.705733 & 1.665756 & 0.214797 & 0.138737 & 1.797348 & 3.614119 & 0.935706 & 2.395806 \\
\hline 9 & 2.705802 & 1.678866 & 0.221835 & 0.140930 & 1.782654 & 3.628950 & 0.943071 & 2.414662 \\
\hline 10 & 2.677496 & 1.612695 & 0.171758 & 0.104031 & 1.865201 & 3.489791 & 0.980518 & 2.244871 \\
\hline 11 & 2.691698 & 1.616866 & 0.182896 & 0.104570 & 1.853477 & 3.529919 & 0.983055 & 2.250678 \\
\hline 12 & 2.700314 & 1.624152 & 0.183897 & 0.105515 & 1.859804 & 3.540824 & 0.987484 & 2.260819 \\
\hline 13 & 2.601492 & 1.542551 & 0.139221 & 0.079315 & 1.870170 & 3.332814 & 0.990556 & 2.094546 \\
\hline 14 & 2.628847 & 1.554005 & 0.165617 & 0.080498 & 1.831203 & 3.426491 & 0.997912 & 2.110099 \\
\hline 15 & 2.652432 & 1.581927 & 0.167527 & 0.083416 & 1.850203 & 3.454662 & 1.015842 & 2.148012 \\
\hline 16 & 2.564242 & 1.518715 & 0.118527 & 0.064069 & 1.889457 & 3.239027 & 1.022602 & 2.014829 \\
\hline 17 & 2.585654 & 1.532091 & 0.126751 & 0.065203 & 1.887853 & 3.283454 & 1.031608 & 2.032574 \\
\hline 18 & 2.590843 & 1.538084 & 0.128757 & 0.065714 & 1.887542 & 3.294144 & 1.035643 & 2.040524 \\
\hline
\end{tabular}




\section{PARAMETER ESTIMATIONS BASED ON KUMARASWAMY DATA}

Table 5. MLEs, their variances and $95 \%$ confidence intervals for parameters using $\alpha=3.00, \theta=5.00$

\begin{tabular}{|c|c|c|c|c|c|c|c|c|}
\hline Schemes & $\hat{\alpha}$ & $\hat{\boldsymbol{\theta}}$ & $V(\hat{\alpha})$ & $V(\hat{\theta})$ & $L L(\hat{\alpha})$ & $U L(\hat{\alpha})$ & $L L(\hat{\theta})$ & $U L(\hat{\theta})$ \\
\hline 1 & 5.516209 & 3.339823 & 0.350879 & 0.743628 & 4.355202 & 6.677216 & 1.649639 & 5.030007 \\
\hline 2 & 5.532574 & 3.379539 & 0.352004 & 0.761419 & 4.369708 & 6.695441 & 1.669256 & 5.089822 \\
\hline 3 & 5.540173 & 3.381127 & 0.356985 & 0.762135 & 4.369107 & 6.711238 & 1.670041 & 5.092213 \\
\hline 4 & 5.484345 & 3.318767 & 0.332414 & 0.611901 & 4.354300 & 6.614389 & 1.785575 & 4.851959 \\
\hline 5 & 5.505484 & 3.325015 & 0.337346 & 0.614207 & 4.367087 & 6.643880 & 1.788936 & 4.861093 \\
\hline 6 & 5.509684 & 3.333368 & 0.343636 & 0.617297 & 4.360723 & 6.658645 & 1.793431 & 4.873306 \\
\hline 7 & 5.312807 & 3.283075 & 0.317312 & 0.538929 & 4.208731 & 6.416883 & 1.844204 & 4.721946 \\
\hline 8 & 5.462259 & 3.311419 & 0.321464 & 0.548275 & 4.350982 & 6.573536 & 1.860126 & 4.762713 \\
\hline 9 & 5.466648 & 3.313941 & 0.322346 & 0.549110 & 4.353848 & 6.579448 & 1.861542 & 4.766339 \\
\hline 10 & 5.241934 & 3.232154 & 0.287640 & 0.417873 & 4.190746 & 6.293123 & 1.965150 & 4.499159 \\
\hline 11 & 5.252454 & 3.250657 & 0.292829 & 0.422671 & 4.191826 & 6.313081 & 1.976400 & 4.524915 \\
\hline 12 & 5.271842 & 3.252974 & 0.308544 & 0.423274 & 4.183126 & 6.360557 & 1.977808 & 4.528140 \\
\hline 13 & 5.189160 & 3.208891 & 0.269755 & 0.343233 & 4.171176 & 6.207144 & 2.060604 & 4.357177 \\
\hline 14 & 5.203086 & 3.220785 & 0.276195 & 0.345782 & 4.173022 & 6.233150 & 2.068242 & 4.373328 \\
\hline 15 & 5.221027 & 3.226427 & 0.277078 & 0.346994 & 4.189318 & 6.252735 & 2.071865 & 4.380989 \\
\hline 16 & 5.160790 & 3.144943 & 0.251366 & 0.274741 & 4.178117 & 6.143463 & 2.117595 & 4.172291 \\
\hline 17 & 5.185568 & 3.147283 & 0.265617 & 0.275150 & 4.175422 & 6.195714 & 2.119170 & 4.175395 \\
\hline 18 & 5.187746 & 3.196740 & 0.266302 & 0.283865 & 4.176298 & 6.199194 & 2.152472 & 4.241009 \\
\hline
\end{tabular}


Table 6. MLEs, their variances and $95 \%$ confidence intervals for parameters using $\alpha=4.00, \theta=2.50$

\begin{tabular}{ccccccccc}
\hline Schemes & $\hat{\boldsymbol{\alpha}}$ & $\hat{\boldsymbol{\theta}}$ & $V(\hat{\boldsymbol{\alpha}})$ & $V(\hat{\boldsymbol{\theta}})$ & $L L(\hat{\alpha})$ & $U L(\hat{\alpha})$ & $L L(\hat{\theta})$ & $U L(\hat{\boldsymbol{\theta}})$ \\
\hline 1 & 4.388710 & 2.724225 & 0.315652 & 0.494760 & 3.287524 & 5.489896 & 1.345577 & 4.102873 \\
2 & 4.419342 & 2.761146 & 0.318780 & 0.508262 & 3.312714 & 5.525969 & 1.363813 & 4.158478 \\
3 & 4.444269 & 2.786841 & 0.325157 & 0.517766 & 3.326626 & 5.561911 & 1.376505 & 4.197178 \\
4 & 4.362571 & 2.681995 & 0.292876 & 0.399617 & 3.301859 & 5.423284 & 1.442977 & 3.921014 \\
5 & 4.366521 & 2.690268 & 0.295321 & 0.402086 & 3.301390 & 5.431652 & 1.447428 & 3.933109 \\
6 & 4.380645 & 2.721175 & 0.309534 & 0.411378 & 3.290184 & 5.471106 & 1.464056 & 3.978294 \\
7 & 4.274244 & 2.667550 & 0.270601 & 0.355791 & 3.254665 & 5.293822 & 1.498445 & 3.836655 \\
8 & 4.315312 & 2.668255 & 0.274710 & 0.355979 & 3.288022 & 5.342602 & 1.498840 & 3.837669 \\
9 & 4.347334 & 2.671510 & 0.281012 & 0.356848 & 3.308326 & 5.386341 & 1.500669 & 3.842352 \\
10 & 4.212643 & 2.589071 & 0.267819 & 0.268132 & 3.198320 & 5.226967 & 1.574155 & 3.603987 \\
11 & 4.242698 & 2.638366 & 0.268059 & 0.278439 & 3.227919 & 5.257478 & 1.604127 & 3.672606 \\
12 & 4.254647 & 2.644628 & 0.270255 & 0.279762 & 3.235719 & 5.273574 & 1.607934 & 3.681322 \\
13 & 4.160297 & 2.574998 & 0.252870 & 0.221021 & 3.174688 & 5.145906 & 1.653547 & 3.496450 \\
14 & 4.198490 & 2.579545 & 0.259322 & 0.221802 & 3.200386 & 5.196594 & 1.656467 & 3.502624 \\
15 & 4.205975 & 2.580193 & 0.266779 & 0.221913 & 3.193622 & 5.218328 & 1.656883 & 3.503503 \\
16 & 4.097057 & 2.552605 & 0.234649 & 0.180994 & 3.147622 & 5.046492 & 1.718754 & 3.386456 \\
17 & 4.106227 & 2.554844 & 0.237447 & 0.181312 & 3.151148 & 5.061306 & 1.720262 & 3.389426 \\
18 & 4.159811 & 2.558519 & 0.239472 & 0.181834 & 3.200668 & 5.118953 & 1.722736 & 3.394302 \\
\hline
\end{tabular}

Tables 1-6 include the maximum likelihood estimates (MLEs), the variances of MLEs, and 95\% confidence intervals for the parameters of the Kumaraswamy distribution under progressively Type II censored samples using different parametric values for various censoring schemes. It has been observed that by increasing the sample size (keeping censoring rate fixed), the estimated value of the parameter become closer to the true value, the variances of the MLEs decrease and widths of $95 \%$ confidence intervals tend to be lesser. This is an indication that the estimators are consistent in nature. It can further be assessed that the censoring schemes, concerned with survivals from the right, result in more precise results than their counterparts. As expected, the increase in true parametric values leads to the slower convergence of the estimates along with larger variances of the 


\section{PARAMETER ESTIMATIONS BASED ON KUMARASWAMY DATA}

estimates which lean to increase the widths of the confidence intervals. The increase in censoring rate, that is, the smaller values of ' $m$ ' has the same natural consequences. However, these negative impacts can be protected by employing larger $(n>30)$ sample sizes.

\section{Conclusion}

This study addressed the problem of estimation of parameters of the Kumaraswamy distribution under progressive censoring based on random removals. The maximum likelihood estimation was used to serve the purpose. The findings of the study indicate that the proposed estimators are consistent in nature. It is interesting to note that the removal of items from the right leads to the most efficient results.

\section{References}

Balakrishnan, N., \& Aggarwala, R. (2000). Progressive Censoring. Theory, Methods and Applications, BirkhÄauser, Boston.

Cohen, A. C. (1963). Progressively censored samples in life testing. Technometrics, 5, 327-33

Kumaraswamy, P. (1980). A generalized probability density function for double-bounded random processes. Journal of Hydrology, 46, 79-88.

Lawless, J. F. (2003). Statistical models and methods for lifetime data. 2nd edition. John Wiley and Sons, Hoboken, 630.

Mann, N. R. (1971). Best linear invariant estimation forWeibull parameter under progressive censoring. Technometrics, 13, 521-534

Meeker, W. Q., \& Escobar, L. A. (1991). Statistical methods for reliability data. New York: John Wiley \& Sons

Ng, H. K. T., Chan, P. S., \& Balakrishnan, N. (2002). Estimation of parameters from progressively censored data using EM algorithm. Computational Statistics and Data Analysis, 39, 371-386.

Ng, H. K. T., Chan, P. S., \& Balakrishnan, N. (2004), Optimal progressive censoring plansfor the Weibull distribution. Technometrics, 46(4), 470-481.

Raqab, M. Z., Asgharzadeh, A., \& Valiollahi, R. (2010). Prediction for Pareto distribution based on progressively Type-II censored samples. Comput. Statist. Data Anal., 54, 1732-1743. 
Soliman, A. A. (2008). Estimation for Pareto model using general progressive censored data and asymmetric loss. Communications in StatisticsTheory and Methods, 37, 1353-1370.

Tse, W., Cersosimo, M. G., \& Gracies, J. M. (2004). Movements disorders and AIDs: a review. Parkinsonisum and related disorders, 10, 323-334.

Viveros, R., \& Balakrishnan, N. (1994). Interval estimation of life characteristics from progressively Censored data. Technometric, 36, 84-91.

Yuen, H. K., \& Tse, S. K. (1996). Parameters estimation for Weibull distributed lifetime under progressive censoring with random removals. Journal of Statistical Computation and Simulation, 55, 57-71. 